\title{
Front Matter: Volume 8864
}

, "Front Matter: Volume 8864," Proc. SPIE 8864, Techniques and Instrumentation for Detection of Exoplanets VI, 886401 (22 October 2013); doi: $10.1117 / 12.2048566$

EDIE Event: SPIE Optical Engineering + Applications, 2013, San Diego, California, SPIE. United States 


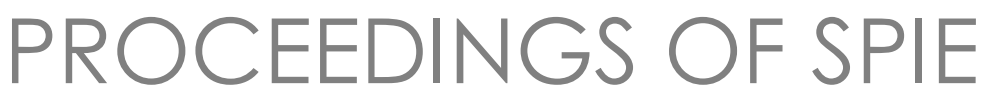

\title{
Techniques and Instrumentation for Detection of Exoplanets VI
}

\author{
Stuart Shaklan
}

Editor

26-29 August 2013

San Diego, California, United States

Sponsored and Published by

SPIE 
The papers included in this volume were part of the technical conference cited on the cover and title page. Papers were selected and subject to review by the editors and conference program committee. Some conference presentations may not be available for publication. The papers published in these proceedings reflect the work and thoughts of the authors and are published herein as submitted. The publisher is not responsible for the validity of the information or for any outcomes resulting from reliance thereon.

Please use the following format to cite material from this book:

Author(s), "Title of Paper," in Techniques and Instrumentation for Detection of Exoplanets VI, edited by Stuart Shaklan, Proceedings of SPIE Vol. 8864 (SPIE, Bellingham, WA, 2013) Article CID Number.

ISSN: 0277-786X

ISBN: 9780819497147

Published by

SPIE

P.O. Box 10, Bellingham, Washington 98227-0010 USA

Telephone +1 3606763290 (Pacific Time) · Fax +1 3606471445

SPIE.org

Copyright (C) 2013, Society of Photo-Optical Instrumentation Engineers.

Copying of material in this book for internal or personal use, or for the internal or personal use of specific clients, beyond the fair use provisions granted by the U.S. Copyright Law is authorized by SPIE subject to payment of copying fees. The Transactional Reporting Service base fee for this volume is $\$ 18.00$ per article (or portion thereof), which should be paid directly to the Copyright Clearance Center (CCC), 222 Rosewood Drive, Danvers, MA 01923. Payment may also be made electronically through CCC Online at copyright.com. Other copying for republication, resale, advertising or promotion, or any form of systematic or multiple reproduction of any material in this book is prohibited except with permission in writing from the publisher. The CCC fee code is 0277-786X/13/\$18.00.

Printed in the United States of America.

Publication of record for individual papers is online in the SPIE Digital Library.

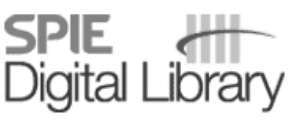

SPIEDigitallibrary.org

Paper Numbering: Proceedings of SPIE follow an e-First publication model, with papers published first online and then in print and on CD-ROM. Papers are published as they are submitted and meet publication criteria. A unique, consistent, permanent citation identifier (CID) number is assigned to each article at the time of the first publication. Utilization of CIDs allows articles to be fully citable as soon as they are published online, and connects the same identifier to all online, print, and electronic versions of the publication. SPIE uses a six-digit CID article numbering system in which:

- The first four digits correspond to the SPIE volume number.

- The last two digits indicate publication order within the volume using a Base 36 numbering

system employing both numerals and letters. These two-number sets start with 00, 01, 02, 03, 04, $05,06,07,08,09,0 A, 0 B \ldots 0 Z$, followed by 10-1Z, 20-2Z, etc.

The CID Number appears on each page of the manuscript. The complete citation is used on the first page, and an abbreviated version on subsequent pages. Numbers in the index correspond to the last two digits of the six-digit CID Number. 


\title{
Contents
}

\author{
xv Conference Committee
}

\section{SESSION 1 PLANETS AND MISSIONS}

886402 Patterns of planet occurrence from doppler and kepler (Invited Paper) [8864-1]

A. W. Howard, Univ. of Hawaii (United States)

886403 Space mission design for exoplanet imaging (Invited Paper) [8864-2]

D. Savransky, Lawrence Livermore National Lab. (United States)

886405 Exoplanet detection and characterization using combined coronagraphy and sub-UAS astrometry from space (Invited Paper) [8864-46]

E. A. Bendek, NASA Ames Research Ctr. (United States); O. Guyon, Steward Observatory, The Univ. of Arizona (United States); R. Belikov, NASA Ames Research Ctr. (United States);

S. M. Ammons, Lawrence Livermore National Lab. (United States); T. Milster, Y.-S. Kim,

L. Johnson, College of Optical Sciences, The Univ. of Arizona (United States)

$886407 \quad$ New Worlds Airship [8864-4]

A. Harness, W. Cash, A. Shipley, Univ. of Colorado at Boulder (United States); T. Glassman,

S. Warwick, Northrop Grumman Aerospace Systems (United States)

886408 Direct imaging of exoplanetary systems with a monolithic multispectral camera [8864-5] B. Hicks, K. Oram, UMLCAR (United States); N. Lewis, Massachusetts Institute of Technology (United States); C. Mendillo, UMLCAR (United States); P. Bierden, Boston Micromachines Corp. (United States); T. Cook, S. Chakrabarti, UMLCAR (United States)

886409 Completion of the EChO phase 0/A study [8864-6]

L. Puig, K. G. Isaak, M. Linder, I. Escudero, P.-E. Crouzet, R. Walker, European Space Agency (Netherlands); P. Eccleston, Rutherford Appleton Lab. (United Kingdom)

8864 OA The Debris Disk Explorer: a balloon-borne coronagraph for observing debris disks [8864-8] L. C. Roberts Jr., G. Bryden, W. Traub, S. Unwin, J. Trauger, J. Krist, J. Aldrich, P. Brugarolas, Jet Propulsion Lab. (United States); K. Stapelfeldt, NASA Goddard Space Flight Ctr. (United States); M. Wyatt, Univ. of Cambridge (United Kingdom); D. Stuchlik, J. Lanzi, NASA Wallops Flight Facility (United States) 
$8864 \mathrm{OB}$ Sphere: complete laboratory performance and prediction for on-sky first light (Invited Paper) [8864-9]

J.-F. Sauvage, ONERA (France) and IPAG, CNRS, Univ. Joseph Fourier (France); J.-L. Beuzit, IPAG, CNRS, Univ. Joseph Fourier (France); R. Roelfsema, NOVA-ASTRON (Netherlands); M. Feldt, Max-Planck-Institut für Astronomie (Germany); K. Dohlen, Lab. d'Astrophysique de Marseille, CNRS, Univ. de Provence (France); D. Mouillet, P. Puget, IPAG, CNRS, Univ. Joseph Fourier (France); F. Wildi, Observatoire de Genève (Switzerland); L. Abe, Lab. Lagrange, CNRS, Observatoire de la Côte d'Azur (France); A. Baruffolo, INAF - Osservatorio Astronomico di Padova (Italy); P. Baudoz, LESIA, CNRS, Observatoire de Paris (France); A. Bazzon, ETH Zürich (Switzerland); A. Boccaletti, T. Buey, LESIA, CNRS, Observatoire de Paris (France); M. Carbillet, Lab. Lagrange, CNRS, Observatoire de la Côte d'Azur (France); J. Charton, IPAG, CNRS, Univ. Joseph Fourier (France); R. Claudi, INAF - Osservatorio Astronomico di Padova (Italy); A. Costille, Lab. d'Astrophysique de Marseille, CNRS, Univ. de Provence (France); A. Delboulbé, IPAG, CNRS, Univ. Joseph Fourier (France); S. Desidera, INAF - Osservatorio Astronomico di Padova (Italy); C. Dominik, Univ. van Amsterdam (Netherlands); M. Downing, European Southern Observatory (Germany); C. Fabron, Lab. d'Astrophysique de Marseille, CNRS, Univ. de Provence (France); P. Feautrier, IPAG, CNRS, Univ. Joseph Fourier (France); E. Fedrigo, European Southern Observatory (Germany); T. Fusco, ONERA (France); E. Giro, INAF - Osservatorio Astronomico di Padova (Italy); L. Gluck, IPAG, CNRS, Univ. Joseph Fourier (France); R. Gratton, INAF - Osservatorio Astronomico di Padova (Italy); T. Henning, Max-PlanckInstitut für Astronomie (Germany); N. Hubin, M. Kasper, European Southern Observatory (Germany); A.-M. Lagrange, IPAG, CNRS, Univ. Joseph Fourier (France); M. Langlois, CRAL, CNRS, Claude Bernard Univ. Lyon 1 (France); D. Le Mignant, Lab. d'Astrophysique de Marseille, CNRS, Univ. de Provence (France); J.-L. Lizon, European Southern Observatory (Germany); F. Madec, Lab. d'Astrophysique de Marseille, CNRS, Univ. de Provence (France); Y. Magnard, IPAG, CNRS, Univ. Joseph Fourier (France); P. Martinez, Lab. d'Astrophysique de Marseille, CNRS, Univ. de Provence (France); D. Mesa, INAF Osservatorio Astronomico di Padova (Italy); O. Möller-Nilsson, Max-Planck-Institut für Astronomie (Germany); T. Moulin, IPAG, CNRS, Univ. Joseph Fourier (France); C. Moutou, Lab. d'Astrophysique de Marseille, CNRS, Univ. de Provence (France); A. Pavlov, Max-Planck-Institut für Astronomie (Germany); D. Perret, LESIA, CNRS, Observatoire de Paris (France); C. Petit, ONERA (France); J. Pragt, NOVA-ASTRON (Netherlands); P. Rabou, S. Rochat, IPAG, CNRS, Univ. Joseph Fourier (France); B. Salasnich, INAF - Osservatorio Astronomico di Padova (Italy); G. Rousset, LESIA, CNRS, Observatoire de Paris (France); H.-M. Schmid, ETH Zürich (Switzerland); A. Sevin, LESIA, CNRS, Observatoire de Paris (France); C. Soenke, European Southern Observatory (Germany); E. Stadler, IPAG, CNRS, Univ. Joseph Fourier (France); M. Suarez, European Southern Observatory (Germany); M. Turatto, INAF - Osservatorio Astronomico di Trieste (Italy); S. Udry, Observatoire de Genève (Switzerland); A. Vigan, Lab. d'Astrophysique de Marseille, CNRS, Univ. de Provence (France); F. Vakili, Lab. Lagrange, CNRS, Observatoire de la Côte d'Azur (France); G. Zins, IPAG, CNRS, Univ. Joseph Fourier (France); A. Zurlo, Lab. d'Astrophysique de Marseille, CNRS, Univ. de Provence (France) 
8864 OC SPHERE-ZIMPOL system testing: status report on polarimetric high contrast results [8864-10] R. Roelfsema, NOVA-ASTRON (Netherlands); D. Gisler, ETH Zürich (Switzerland); J. Pragt, NOVA-ASTRON (Netherlands); H. M. Schmid, A. Bazzon, ETH Zürich (Switzerland); C. Dominik, Astronomical Institute "Anton Pannekoek" (Netherlands); A. Baruffolo, INAF - Osservatorio Astronomico di Padova (Italy); A. Boccaletti, LESIA - Observatoire de Paris (France); J.-L. Beuzit, IPAG, CNRS, Univ. Joseph Fourier (France); A. Costille, K. Dohlen, Lab. d'Astrophysique de Marseille, CNRS, Univ. de Provence (France); M. Downing, European Southern Observatory (Germany); E. Elswijk, M. de Haan, NOVA-ASTRON (Netherlands); N. Hubin, M. Kasper, European Southern Observatory (Germany); C. Keller, M. Kenworthy, Leiden Observatory (Netherlands); J.-L. Lizon, European Southern Observatory (Germany); P. Martinez, D. Mouillet, IPAG, CNRS, Univ. Joseph Fourier (France); A. Pavlov, Max-PlanckInstitut fur Astronomie (Germany); P. Puget, IPAG, CNRS, Univ. Joseph Fourier (France); B. Salasnich, INAF - Osservatorio Astronomico di Padova (Italy); J.-F. Sauvage, ONERA (France); C. Thalmann, Astronomical Institute "Anton Pannekoek" (Netherlands); F. Wildi, Univ. de Geneve (Switzerland)

\section{SESSION 3 GROUND-BASED EXOPLANET DETECTION AND CHARACTERIZATION II}

8864 OD Experimental validation of the high-order coronagraphic phase diversity (COFFEE) on the SPHERE system [8864-11]

B. Paul, ONERA (France), Lab. d'Astrophysique de Marseille, Univ. Aix-Marseille (France) and Groupement d'intérêt scientifique PHASE (France); J.-F. Sauvage, ONERA (France), IPAG, CNRS, Univ. Joseph Fourier (France) and Groupement d'intérêt scientifique PHASE (France); L. M. Mugnier, ONERA (France) and Groupement d'intérêt scientifique PHASE (France); K. Dohlen, Lab. d'Astrophysique de Marseille, CNRS, Univ. Aix-Marseille (France) and Groupement d'intérêt scientifique PHASE (France); D. Mouillet, IPAG, CNRS, Univ. Joseph Fourier (France) and Groupement d'intérêt scientifique PHASE (France); T. Fusco, ONERA (France), Lab. d'Astrophysique de Marseille, Univ. Aix-Marseille (France) and Groupement d'intérêt scientifique PHASE (France); J.-L. Beuzit, IPAG, CNRS, Univ. Joseph Fourier (France) and Groupement d'intérêt scientifique PHASE (France); M. N'Diaye, Space Telescope Science Institute (United States) and Groupement d'intérêt scientifique PHASE (France); M. Ferrari, Lab. d'Astrophysique de Marseille, CNRS, Univ. Aix-Marseille (France) and Groupement d'intérêt scientifique PHASE (France) 
8864 OE ESPRESSO, an exo-Earths hunter for the VLT [8864-12]

D. Mégevand, Observatoire de Genève (Switzerland); F. M. Zerbi, INAF - Osservatorio Astronomico di Brera (Italy); P. Di Marcantonio, INAF - Osservatorio Astronomico di Trieste (Italy); A. Cabral, Univ. de Lisboa (Portugal); F. Pepe, Observatoire de Genève (Switzerland); S. Cristiani, INAF - Osservatorio Astronomico di Trieste (Italy); R. Rebolo, Instituto de Astrofísica de Canarias (Spain); N. C. Santos, Univ. do Porto (Portugal); H. Dekker, European Southern Observatory (Germany); M. Abreu, Univ. de Lisboa (Portugal); M. Affolter, Univ. Bern (Switzerland); M. Aliverti, INAF - Osservatorio Astronomico di Brera (Italy); M. Amate, Instituto de Astrofísica de Canarias (Spain); G. Avila, European Southern Observatory (Germany); V. Baldini, INAF - Osservatorio Astronomico di Trieste (Italy); P. Bristow, European Southern Observatory (Germany); C. Broeg, Univ. Bern (Switzerland); R. Cirami, INAF - Osservatorio Astronomico di Trieste (Italy); J. Coelho, Univ. de Lisboa (Portugal); P. Conconi, INAF - Osservatorio Astronomico di Brera (Italy); I. Coretti, G. Cupani, V. D'Odorico, INAF - Osservatorio Astronomico di Trieste (Italy); V. De Caprio, INAF - Osservatorio Astronomico di Brera (Italy); B. Delabre, R. Dorn, European Southern Observatory (Germany); P. Figueira, Univ. do Porto (Portugal); A. Fragoso, Instituto de Astrofísica de Canarias (Spain); S. Galeotta, INAF - Osservatorio Astronomico di Trieste (Italy); L. Genolet, Observatoire de Genève (Switzerland); R. Gomes, LOLS, Univ. de Lisboa (Portugal); J. Gonzalez Hernandez, Instituto de Astrofísica de Canarias (Spain); I. Hughes, Observatoire de Genève (Switzerland); O. Iwert, F. Kerber, European Southern Observatory (Germany); M. Landoni, INAF - Osservatorio Astronomico di Brera (Italy); J.-L. Lizon, European Southern Observatory (Germany); C. Lovis, C. Maire, Observatoire de Genève (Switzerland); M. Mannetta, INAF - Osservatorio Astronomico di Trieste (Italy); C. Martins, Univ. do Porto (Portugal); P. Molaro, INAF - Osservatorio Astronomico di Trieste (Italy); M. Monteiro, Univ. do Porto (Portugal); A. Oliveira, LOLS, Univ. de Lisboa (Portugal); M. R. Zapatero Osorio, Instituto Nacional de Técnica Aeroespacial (Spain); E. Poretti, INAF Osservatorio Astronomico di Brera (Italy); J. L. Rasilla, Instituto de Astrofísica de Canarias (Spain); M. Riva, INAF - Osservatorio Astronomico di Brera (Italy); S. Santana Tschudi, Instituto de Astrofísica de Canarias (Spain); P. Santos, LOLS, Univ. de Lisboa (Portugal); D. Sosnowska, Observatoire de Genève (Switzerland); S. Sousa, Univ. do Porto (Portugal); F. Tenegi, Instituto de Astrofísica de Canarias (Spain); G. Toso, INAF - Osservatorio Astronomico di Brera (Italy); E. Vanzella, M. Viel, INAF - Osservatorio Astronomico di Trieste (Italy)

8864 OF ESPRESSO front end exposure meter: a chromatic approach to radial velocity correction [8864-13]

M. Landoni, Univ. degli Studi dell'Insubria (Italy) and INAF - Osservatorio Astronomico di Brera (Italy); M. Riva, INAF - Osservatorio Astronomico di Brera (Italy); F. Pepe, Observatoire de Genève (Switzerland); P. Conconi, F. M. Zerbi, INAF - Osservatorio Astronomico di Brera (Italy); A. Cabral, Univ. de Lisboa (Portugal); S. Cristiani, INAF - Osservatorio Astronomico di Trieste (Italy); D. Mégevand, Observatoire de Genève (Switzerland) 
8864 OG Precision near-infrared radial velocity instrumentation II: noncircular core fiber scrambler [8864-14]

P. P. Plavchan, NASA Exoplanet Science Institute, California Institute of Technology (United States); M. Bottom, P. Gao, California Institute of Technology (United States); J. K. Wallace, B. Mennesson, Jet Propulsion Lab. (United States); D. Ciardi, NASA Exoplanet Science Institute, California Institute of Technology (United States); S. Crawford, S. Lin, Jet Propulsion Lab. (United States); C. Beichman, C. Brinkworth, NASA Exoplanet Science Institute, California Institute of Technology (United States); J. Johnson, Harvard Univ. (United States); C. Davison, R. White, Georgia State Univ. (United States); G. Anglada-Escude, Univ. Göttingen (Germany); K. von Braun, Max-Planck-Institut für Astronomie (Germany); G. Vasisht, Jet Propulsion Lab. (United States); L. Prato, Lowell Observatory (United States); S. Kane, San Francisco State Univ. (United States); A. Tanner, Mississippi State Univ. (United States); B. Walp, SOFIA (United States); S. Mills, The Univ. of Chicago (United States)

$8864 \mathrm{OH}$ Design of the CHARIS integral field spectrograph for exoplanet imaging [8864-15] T. D. Groff, M. A. Peters, N. J. Kasdin, G. Knapp, M. Galvin, M. A. Carr, Princeton Univ. (United States); M. W. McElwain, NASA Goddard Space Flight Ctr. (United States); T. Brandt, M. Janson, J. E. Gunn, R. Lupton, Princeton Univ. (United States); O. Guyon, F. Martinache, N. Jovanovic, Subaru Telescope, National Astronomical Observatory of Japan (United States); M. Hayashi, National Astronomical Observatory of Japan (Japan); N. Takato,

Subaru Telescope, National Astronomical Observatory of Japan (United States)

SESSION 4 GROUND-BASED EXOPLANET DETECTION AND CHARACTERIZATION III

8864 Ol Small-angle, high-contrast exoplanet imaging with the L-band AGPM vector vortex coronagraph now offered at the VLT [8864-16]

D. Mawet, European Southern Observatory (Chile) and Jet Propulsion Lab. (United States); O. Absil, Univ. de Liège (Belgium); J. Milli, European Southern Observatory (Chile) and IPAG, CNRS, Univ. Joseph Fourier (France); C. Delacroix, Univ. de Liège (Belgium); J. H. Girard, J. O'Neal, European Southern Observatory (Chile); P. Baudoz, LESIA - Observatoire de Paris (France); A. Boccaletti, Univ. de Liège (Belgium) P. Bourget, European Southern Observatory (Chile); P. Forsberg, Uppsala Univ. (Sweden); F. Gonté, European Southern Observatory (Chile); S. Habraken, European Southern Observatory (Chile); M. Karlsson, Uppsala Univ. (Sweden); M. Kasper, European Southern Observatory Headquarters (Germany); A.-M. Lagrange, IPAG, CNRS, Univ. Joseph Fourier (France); J.-L. Lizon, K. Muzic, E. Peña, European Southern Observatory (Chile); R. Olivier, GDTech s.a., LIEGE Science Park (Belgium); N. Slusarenko, European Southern Observatory (Chile); L. E. Tacconi-Garman, European Southern Observatory Headquarters (Germany); J. Surdej, Univ. de Liège (Belgium)

$88640 \mathrm{~J}$ Adaptive phase-mask coronagraph with amplitude and phase modulation for high dynamic range synchronous detection: APM $^{2}$ coronagraph [8864-17]

P. Bourget, D. Mawet, P. Mardones, N. Schuhler, European Southern Observatory (Chile);

L. Pueyo, Space Telescope Science Institute (United States); J. Girard, P. Haguenaver,

F. Gonte, European Southern Observatory (Chile) 
8864 OK Electric field conjugation with the project 1640 coronagraph [8864-19]

E. Cady, Jet Propulsion Lab. (United States); C. Baranec, California Institute of Technology

(United States); C. Beichman, Jet Propulsion Lab. (United States), California Institute of Technology (United States) and NASA Exoplanet Science Institute (United States);

D. Brenner, American Museum of Natural History (United States); R. Burruss, Jet Propulsion Lab. (United States); J. Crepp, Notre Dame Univ. (United States); R. Dekany, D. Hale, L. Hillenbrand, S. Hinkley, California Institute of Technology (United States); E. R. Ligon, T. Lockhart, Jet Propulsion Lab. (United States); B. R. Oppenheimer, American Museum of Natural History (United States); I. Parry, Cambridge Univ. (United Kingdom); L. Pueyo, Space Telescope Science Institute (United States); E. Rice, American Museum of Natural History (United States) and College of Staten Island (United States); L. C. Roberts Jr., J. Roberts, M. Shao, Jet Propulsion Lab. (United States); A. Sivaramakrishnan, R. Soummer, Space Telescope Science Institute (United States); H. Tang, T. Truong, G. Vasisht, F. Vescelus, J. K. Wallace, C. Zhai, Jet Propulsion Lab. (United States); N. Zimmerman, Max-PlanckInstitut für Astronomie (Germany)

8864 OL Estimate low- and high-order wavefront using P1640 calibrator measurements [8864-20] C. Zhai, G. Vasisht, M. Shao, T. Lockhart, E. Cady, Jet Propulsion Lab. (United States); B. Oppenheimer, American Museum of Natural History (United States); R. Burruss, J. Roberts, Jet Propulsion Lab. (United States); C. Beichman, California Institute of Technology (United States); D. Brenner, American Museum of Natural History (United States); J. Crepp, Univ. of Notre Dame (United States); R. Dekany, L. Hillenbrand, S. Hinkley, California Institute of Technology (United States); I. Parry, Cambridge Univ. (United Kingdom); L. Pueyo, Johns Hopkins Univ. (United States); E. Rice, American Museum of Natural History (United States); L. C. Roberts Jr., Jet Propulsion Lab. (United States); A. Sivaramakrishnan, Space Telescope Science Institute (United States); R. Soummer, Johns Hopkins Univ. (United States); H. Tang, F. Vescelus, K. Wallace, Jet Propulsion Lab. (United States); N. Zimmerman, Max-PlanckInstitut für Astronomie (Germany)

\section{SESSION 5 HIGH-CONTRAST IMAGING TECHNOLOGY AND MODELING I}

8864 OM High-contrast imaging with a self-coherent camera (Invited Paper) [8864-21]

R. Galicher, Univ. Paris 7-Denis Diderot (France) and LESIA, CNRS, Observatoire de Paris (France); J. Mazoyer, Univ. Paris 7-Denis Diderot (France); P. Baudoz, LESIA, CNRS, Observatoire de Paris; G. Rousset, Univ. Paris 7-Denis Diderot (France) and LESIA, CNRS, Observatoire de Paris (France)

$88640 \mathrm{~N}$ Speckle correction in polychromatic light with the self-coherent camera for the direct detection of exoplanets [8864-22]

J. Mazoyer, R. Galicher, P. Baudoz, G. Rousset, LESIA, CNRS, Observatoire de Paris (France)

886400 Experimental study of the low-order wavefront sensor for the high-contrast coronagraphic imager EXCEDE [8864-23]

J. Lozi, The Univ. of Arizona (United States); R. Belikov, NASA Ames Research Ctr. (United

States); G. Schneider, O. Guyon, The Univ. of Arizona (United States); E. Pluzhnik, S. J. Thomas, NASA Ames Research Ctr. (United States); F. Martinache, Subaru Telescope, National Astronomical Observatory of Japan (United States) 
$8864 \mathrm{OP}$ Assessing the performance limits of internal coronagraphs through end-to-end modeling [8864-24]

J. E. Krist, Jet Propulsion Lab. (United States); R. Belikov, NASA Ames Research Ctr. (United States); L. Pueyo, Space Telescope Science Institute (United States); D. P. Mawet, European Southern Observatory (Chile); D. Moody, J. T. Trauger, S. B. Shaklan, Jet Propulsion Lab. (United States)

$8864 \mathrm{OQ}$ HCIT contrast performance sensitivity studies: simulation versus experiment [8864-25] E. Sidick, S. Shaklan, J. Krist, E. J. Cady, B. Kern, K. Balasubramanian, Jet Propulsion Lab. (United States)

8864 OR Laboratory demonstration of Phase Induced Amplitude Apodization (PIAA) coronagraph with better than $10^{-9}$ contrast [8864-26]

B. Kern, Jet Propulsion Lab. (United States); O. Guyon, Steward Observatory, The Univ. of Arizona (United States); A. Kuhnert, A. Niessner, Jet Propulsion Lab. (United States);

F. Martinache, Steward Observatory, The Univ. of Arizona (United States);

K. Balasubramanian, Jet Propulsion Lab. (United States)

\section{SESSION 6 HIGH-CONTRAST IMAGING TECHNOLOGY AND MODELING II}

8864 OS Image analysis with speckles altered by a deformable mirror [8864-27]

E. J. Young, N. J. Kasdin, A. Carlotti, Princeton Univ. (United States)

8864 OT Demonstration of symmetric dark holes using two deformable mirrors at the high-contrast imaging testbed [8864-28]

A. J. E. Riggs, T. D. Groff, A. Carlotti, N. J. Kasdin, Princeton Univ. (United States); E. J. Cady,

B. D. Kern, A. Kuhnert, Jet Propulsion Lab. (United States)

8864 OU MEMS deformable mirror CubeSat testbed [8864-29]

K. L. Cahoy, A. D. Marinan, B. Novak, C. Kerr, T. Nguyen, M. Webber, G. Falkenburg,

A. Barg, K. Berry, A. Carlton, Massachusetts Institute of Technology (United States);

R. Belikov, E. A. Bendek, NASA Ames Research Ctr. (United States)

8864 OV Planar waveguide integrated spatial filter array [8864-30]

J. Ai, F. Dimov, Luminit LLC (United States); R. Lyon, NASA Goddard Space Flight Ctr.

(United States); N. Rakuljic, C. Griffo, X. Xia, E. Arik, Luminit LLC (United States)

\section{SESSION 7 HIGH-CONTRAST IMAGING TECHNOLOGY AND MODELING III}

8864 OW EXCEDE technology development II: demonstration of high contrast at 1.2 $\mathrm{\lambda} / \mathrm{D}$ and preliminary broadband results [8864-31]

R. Belikov, E. Bendek, T. P. Greene, NASA Ames Research Ctr. (United States); O. Guyon, J. Lozi, The Univ. of Arizona (United States); D. H. Lynch, K. E. Newman, E. Pluzhnik, NASA Ames Research Ctr. (United States); G. Schneider, The Univ. of Arizona (United States);

D. Tenerelli, Lockheed Martin Space Systems Co. (United States); S. J. Thomas,

F. C. Witteborn, NASA Ames Research Ctr. (United States) 
$88640 \mathrm{X}$ Improving image contrast for the direct detection of exoplanets at small inner working angles [8864-18]

S. Thomas, E. Pluzhnik, J. Lozi, R. Belikov, F. Witteborn, T. Greene, NASA Ames Research Ctr. (United States); G. Schneider, O. Guyon, The Univ. of Arizona (United States)

8864 OY High-contrast imaging results with the vortex coronagraph [8864-32]

E. Serabyn, J. Trauger, D. Moody, Jet Propulsion Lab. (United States); D. Mawet, European Southern Observatory (Chile); K. Liewer, J. Krist, B. Kern, Jet Propulsion Lab. (United States)

\section{SESSION 8 AFTA TECHNOLOGY}

$88640 Z$ High-contrast imaging with an arbitrary aperture: active correction of aperture discontinuities (Invited Paper) [8864-33]

L. Pueyo, Space Telescope Science Institute (United States); C. Norman, Johns Hopkins Univ. (United States); R. Soummer, M. Perrin, M. N'Diaye, E. Choquet, Space Telescope Science Institute (United States)

886410 Shaped pupil coronagraphy with WFIRST-AFTA [8864-34]

A. Carlotti, Princeton Univ. (United States) and IPAG, CNRS, Univ. Joseph-Fourier (France); N. J. Kasdin, R. J. Vanderbei, Princeton Univ. (United States)

886411 The multistage and ring-apodized vortex coronagraph: two simple, small-angle coronagraphic solutions for heavily obscured apertures [8864-35]

D. Mawet, European Southern Observatory (Chile) and Jet Propulsion Lab. (United States); L. Pueyo, Space Telescope Science Institute (United States); A. Carlotti, Princeton Univ. (United States) and IPAG, CNRS, Univ. Joseph Fourier (France); B. Mennesson, E. Serabyn, J. Wallace, Jet Propulsion Lab. (United States); P. Baudoz, LESIA, CNRS, Observatoire de Paris (France) and Univ. Paris 7-Denis Diderot (France)

886412 Complex apodized Lyot coronagraph for exoplanet imaging with partially obscured telescope apertures [8864-36]

J. Trauger, D. Moody, B. Gordon, Jet Propulsion Lab. (United States)

886413 Wavefront control scenarios for a coronagraph on an AFTA-like space telescope [8864-37] T. D. Groff, N. J. Kasdin, Princeton Univ. (United States); S. Shaklan, Jet Propulsion Lab. (United States); L. Pueyo, Space Telescope Science Institute (United States)

886414 Imaging Earth-like planets around late-type stars with low-inner working angle PIAA coronagraphy [8864-38]

O. Guyon, Steward Observatory, The Univ. of Arizona (United States) and Subaru Telescope, National Astronomical Observatory of Japan (United States)

886415 The AFTA coronagraph instrument [8864-39]

S. Shaklan, M. Levine, M. Foote, Jet Propulsion Lab. (United States); M. Rodgers, Synopsys, Inc. (United States); M. Underhill, L. Marchen, D. Klein, Jet Propulsion Lab. (United States) 
886417 Verifying occulter deployment tolerances as part of NASA's technology development for exoplanet missions [8864-41]

N. J. Kasdin, Princeton Univ. (United States); D. Lisman, S. Shaklan, M. Thomson, D. Webb, E. Cady, Jet Propulsion Lab. (United States); G. W. Marks, A. Lo, Northrop Grumman Aerospace Systems (United States)

886418 Achieving high-contrast ratios with a 60-cm starshade [8864-42]

T. Glassman, S. Casement, S. Warwick, O. Armagan, J. Donovan, Northrop Grumman Aerospace Systems (United States)

886419 Progress on optical verification for occulter-based high contrast imaging [8864-43] D. Sirbu, N. J. Kasdin, R. J. Vanderbei, Princeton Univ. (United States)

8864 lA Starshade optical edge modeling, requirements, and laboratory tests [8864-44] S. R. Martin, S. Shaklan, S. Crawford, Jet Propulsion Lab. (United States); S.-C. Lee, Applied Sciences Lab., Inc. (United States); B. Khayatian, D. Hoppe, E. Cady, P. D. Lisman, Jet Propulsion Lab. (United States)

\section{SESSION 10 ASTROMETRY}

8864 1B Astrometric detection of exoplanets from the ground (Invited Paper) [8864-45] J. Sahlmann, Observatoire de Genève (Switzerland); P. F. Lazorenko, Main Astronomical Observatory (Ukraine); A. Mérand, European Southern Observatory (Chile); D. Queloz, D. Ségransan, Observatoire de Genève (Switzerland); J. Woillez, European Southern Observatory (Germany)

8864 1C First experimental results of very high accuracy centroiding measurements for the neat astrometric mission [8864-47]

A. Crouzier, F. Malbet, O. Preis, F. Henault, P. Kern, G. Martin, P. Feautrier, E. Stadler, S. Lafrasse, A. Delboulbé, E. Behar, M. Saint-Pe, J. Dupont, S. Potin, IPAG, CNRS, Univ. Joseph Fourier (France); C. Cara, M. Donati, E. Doumayrou, P. O. Lagage, Commissariat à I'Énergie Atomique et aux Energies Altlernatives (France); A. Léger, Institut d'Astrophysique Spatiale, Ctr. Univ. d'Orsa (France); J. M. LeDuigou, Ctr. National d'Études Spatiales (France); M. Shao, R. Goullioud, Jet Propulsion Lab. (United States)

8864 ID NEAT: an astrometric mission to detect nearby planetary systems down to the Earth mass [8864-48]

F. Malbet, A. Crouzier, IPAG, CNRS-INSU, Univ. Joseph Fourier (France); A. Léger, IAS, CNRSINSU, Univ. Paris Sud (France); M. Shao, R. Goullioud, Jet Propulsion Lab. (United States); P. O. Lagage, Lab. AIM, CEA-IRFU, CNRS-INSU, Univ. Paris 7-Denis Diderot (France); M. Delpech, Ctr. National d'Études Spatiales (France) and the NEAT/microNEAT consortium (France) 
$8864 \mathrm{lF}$ Survey of experimental results in high-contrast imaging for future exoplanet missions [8864-50]

P. R. Lawson, Jet Propulsion Lab. (United States); R. Belikov, NASA Ames Research Ctr. (United States); W. Cash, Univ. of Colorado at Boulder (United States); M. Clampin, NASA Goddard Space Flight Ctr. (United States); T. Glassman, Northrop Grumman Aerospace Systems (United States); O. Guyon, Steward Observatory, The Univ. of Arizona (United States); N. J. Kasdin, Princeton Univ. (United States); B. D. Kern, Jet Propulsion Lab. (United States); R. Lyon, NASA Goddard Space Flight Ctr. (United States); D. Mawet, European Southern Observatory (Chile); D. Moody, Jet Propulsion Lab. (United States); R. Samuele, Northrop Grumman Aerospace Systems (United States); E. Serabyn, Jet Propulsion Lab. (United States); D. Sirbu, Princeton Univ. (United States); J. Trauger, Jet Propulsion Lab. (United States)

8864 IG Achromatic focal plane mask for exoplanet imaging coronagraphy [8864-51] K. Newman, The Univ. of Arizona (United States) and NASA Ames Research Ctr. (United States); R. Belikov, NASA Ames Research Ctr. (United States); O. Guyon, The Univ. of Arizona (United States); K. Balasubramanian, D. Wilson, Jet Propulsion Lab. (United States)

$8864 \mathrm{lH} \quad$ Pressure and temperature stabilization of an existing Echelle spectrograph IV [8864-52] A. Brucalassi, F. Grupp, Max-Planck-Institut für extraterrestrische Physik (Germany) and Univ. Observatory München (Germany); F. Lang, Univ. Observatory München (Germany); L. Wang, National Astronomical Observatories of China (China); C. Franik, H. Kellerm, Univ. Observatory München (Germany); S. M. Hu, Shandong Univ. at Weihai (China); U. Hopp, Univ. Observatory München (Germany); R. Bender, Max-Planck-Institut für extraterrestrische Physik (Germany) and Univ. Observatory München (Germany)

$88641 \mathrm{~J}$ Precision near-infrared radial velocity instrumentation I: absorption gas cells [8864-54] P. P. Plavchan, NASA Exoplanet Science Institute (United States); G. Anglada-Escude, Univ. Göttingen (Germany); R. White, Georgia State Univ. (United States); P. Gao, California Institute of Technology (United States); C. Davison, Georgia State Univ. (United States); S. Mills, The Univ. of Chicago (United States); C. Beichman, C. Brinkworth, NASA Exoplanet Science Institute (United States); J. Johnson, Harvard Univ. (United States); M. Bottom, California Institute of Technology (United States); D. Ciardi, NASA Exoplanet Science Institute (United States); K. Wallace, B. Mennesson, Jet Propulsion Lab. (United States); K. von Braun, Max-Planck-Institut für Astronomie (Germany); G. Vasisht, Jet Propulsion Lab. (United States); L. Prato, Lowell Observatory (United States); S. Kane, San Francisco State Univ. (United States); A. Tanner, Mississippi State Univ. (United States); B. Walp, SOFIA (United States); S. Crawford, S. Lin, Jet Propulsion Lab. (United States)

$88641 \mathrm{~K}$ High-contrast imager for complex aperture telescopes (HiCAT): 1. testbed design [8864-55]

M. N'Diaye, E. Choquet, Space Telescope Science Institute (United States); L. Pueyo, Space Telescope Science Institute (United States) and Johns Hopkins Univ. (United States); E. Elliot, M. D. Perrin, Space Telescope Science Institute (United States); J. K. Wallace, Jet Propulsion Lab. (United States); T. Groff, Princeton Univ. (United States); A. Carlotti, IPAG, CNRS, Univ. Joseph Fourier (France); D. Mawet, European Southern Observatory (Chile) and Jet Propulsion Lab. (United States); M. Sheckells, Johns Hopkins Univ. (United States); S. Shaklan, Jet Propulsion Lab. (United States); B. Macintosh, Lawrence Livermore National Lab. (Canada); N. J. Kasdin, Princeton Univ. (United States); R. Soummer, Space Telescope Science Institute (United States) 
$8864 \mathrm{lL} \quad$ Flat field errors and intra-pixel sensitivities for non-redundant aperture masking interferometry on JWST NIRISS [8864-56]

A. Z. Greenbaum, Johns Hopkins Univ. (United States); A. Sivaramakrishnan, Space Telescope Science Institute (United States); L. Pueyo, Johns Hopkins Univ. (United States) and Space Telescope Science Institute (United States)

$88641 \mathrm{M}$ Could Jean-Dominique Cassini see the famous division in Saturn's rings? [8864-57] J. Lozi, The Univ. of Arizona (United States), Ctr. National d'Études Spatiales (France) and Onera (France); J.-M. Reess, A. Semery, E. Lhomé, S. Jacquinod, M. Combes, P. Bernardi, R. Andretta, M. Motisi, LESIA - Observatoire de Paris (France); L. Bobis, E. Kaftan, Observatoire de Paris (France)

8864 IN The optical design of CHARIS: an exoplanet IFS for the Subaru telescope [8864-58] M. A. Peters-Limbach, T. D. Groff, N. J. Kasdin, Princeton Univ. (United States); D. Driscoll, L-3 Communications IOS-SSG (United States); M. Galvin, Princeton Univ. (United States); A. Foster, The Pennsylvania State Univ. (United States); M. A. Carr, Princeton Univ. (United States); D. LeClerc, R. Fagan, L-3 Communications IOS-SSG (United States); M. W. McElwain, NASA Goddard Space Flight Ctr. (United States); G. Knapp, T. Brandt, M. Janson, Princeton Univ. (United States); O. Guyon, N. Jovanovic, F. Martinache, M. Hayashi, N. Takato, Subaru Telescope, National Astronomical Observatory of Japan (United States)

886410 PISCES: an integral field spectrograph to advance high contrast imaging technologies [8864-59]

M. W. McElwain, NASA Goddard Space Flight Ctr. (United States); M. D. Perrin, Space Telescope Science Institute (United States); Q. Gong, NASA Goddard Space Flight Ctr. (United States); A. N. Wilkins, NASA Goddard Space Flight Ctr. (United States) and Univ. of Maryland, College Park (United States); K. R. Stapelfeldt, B. E. Woodgate, NASA Goddard Space Flight Ctr. (United States); T. D. Brandt, Princeton Univ. (United States); S. R. Heap, NASA Goddard Space Flight Ctr. (United States); G. M. Hilton, NASA Goddard Space Flight Ctr. (United States) and Universities Space Research Association (United States); J. W. Kruk, NASA Goddard Space Flight Ctr. (United States); D. Moody, J. Trauger, Jet Propulsion Lab. (United States)

8864 IP Optimal apodizers for the vector vortex coronagraph with on-axis telescopes [8864-60] A. Carlotti, Princeton Univ. (United States) and Univ. Joseph Fourier (France); D. Mawet, European Southern Observatory (Chile); L. Pueyo, Space Telescope Science Institute (United States)

8864 IQ Hybrid coronagraphic design: optimization of complex apodizers [8864-61]

A. Carlotti, Princeton Univ. (United States) and IPAG, CNRS, Univ. Joseph Fourier (France); N. J. Kasdin, R. J. Vanderbei, A. J. E. Riggs, Princeton Univ. (United States)

8864 IR High contrast internal and external coronagraph masks produced by various techniques [8864-62]

K. Balasubramanian, D. Wilson, V. White, R. Muller, M. Dickie, K. Yee, R. Ruiz, S. Shaklan, E. Cady, B. Kern, Jet Propulsion Lab. (United States); R. Belikov, NASA Ames Research Ctr. (United States); O. Guyon, The Univ. of Arizona (United States); N. J. Kasdin, Princeton Univ. (United States) 
8864 is HOMES Holographic Optical Method for Exoplanet Spectroscopy [8864-63]

T. D. Ditto, 3DeWitt LLC (United States); S. P. McGrew, New Light Industries, Ltd. (United States)

8864 IT Holographic spectrograph for space telescope [8864-69]

T. D. Ditto, 3DeWitt LLC (United States); S. Lysenko, Univ. de Puerto Rico Mayagüez (United States); M. Crenshaw, Holographic Design (Canada)

8864 IV Wavelength calibration and closure phases with the Gemini Planet Imager IFS using its non-redundant mask [8864-66]

A. Z. Greenbaum, Johns Hopkins Univ. (United States); A. Sivaramakrishnan, L. Pueyo, Space Telescope Science Institute (United States); P. Ingraham, Univ. de Montréal (Canada); S. Thomas, NASA Ames Research Ctr. (United States); S. Wolff, Johns Hopkins Univ. (United States); M. D. Perrin, Space Telescope Science Institute (United States); B. Norris, P. G. Tuthill, The Univ. of Sydney (Australia)

8864 IW Aqueye+: a wavefront sensorless adaptive optics system for narrow field coronagraphy [8864-67]

E. Verroi, G. Naletto, C. Barbieri, M. Zaccariotto, Univ. degli Studi di Padova (Italy);

T. Occhipinti, I. Capraro, Adaptica S.r.I. (Italy); L. Zampieri, INAF - Osservatorio Astronomico di Padova (Italy); A. Cardullo, Adaptica S.r.l. (Italy); F. Frassetto, Istituto di Fotonica e Nanotecnologie, CNR (Italy); L. Brugiolo, F. Romanato, F. Ricci, M. Gintoli, Univ. degli Studi di Padova (Italy)

8864 IY Comparison of simulated contrast performance of different Phase Induced Amplitude Apodization (PIAA) coronagraph configurations [8864-70]

E. Sidick, B. Kern, A. Kuhnert, S. Shaklan, Jet Propulsion Lab. (United States)

886412 Spectral flattening of supercontinua with a spatial light modulator [8864-71]

R. A. Probst, Max-Planck-Institut für Quantenoptik (Germany); T. Steinmetz, Max-PlanckInstitut für Quantenoptik (Germany) and Menlo Systems GmbH (Germany); T. Wilken, MaxPlanck-Institut für Quantenoptik (Germany); G. K. L. Wong, Max-Planck-Institut für die Physik des Lichts (Germany); H. Hundertmark, Menlo Systems GmbH (Germany); S. P. Stark, P. St. J. Russell, Max-Planck-Institut für die Physik des Lichts (Germany); T. W. Hänsch, MaxPlanck-Institut für Quantenoptik (Germany); R. Holzwarth, Max-Planck-Institut für Quantenoptik (Germany) and Menlo Systems GmbH (Germany); T. Udem, Max-PlanckInstitut für Quantenoptik (Germany)

Author Index

xiv 


\title{
Conference Committee
}

\author{
Program Track Chair
}

Oswald H. Siegmund, University of California, Berkeley (United States)

Conference Chair

Stuart Shaklan, Jet Propulsion Laboratory (United States)

\section{Conference Program Committee}

Olivier Guyon, Subaru Telescope, National Astronomical Observatory of Japan (United States), Research Corporation of University of Hawaii (United States) and The University of Arizona (United States)

Bruce A. Macintosh, Lawrence Livermore National Laboratory (United States)

Fabien Malbet, Laboratoire d'Astrophysique de Grenoble (France)

Dimitri P. Mawet, European Southern Observatory (Chile)

M. Charley Noecker, Jet Propulsion Laboratory (United States)

Rémi Soummer, Space Telescope Science Institute (United States)

Session Chairs

1 Planets and Missions

M. Charley Noecker, Jet Propulsion Laboratory (United States)

2 Ground-based Exoplanet Detection and Characterization I

M. Charley Noecker, Jet Propulsion Laboratory (United States)

3 Ground-based Exoplanet Detection and Characterization II

Olivier Guyon, Subaru Telescope, National Astronomical Observatory of Japan (United States)

4 Ground-based Exoplanet Detection and Characterization III

Olivier Guyon, Subaru Telescope, National Astronomical Observatory of Japan (United States)

5 High-contrast Imaging Technology and Modeling I

Dimitri Mawet, European Southern Observatory (Chile)

6 High-contrast Imaging Technology and Modeling II

Dimitri Mawet, European Southern Observatory (Chile)

7 High-contrast Imaging Technology and Modeling III

Dimitri Mawet, European Southern Observatory (Chile) 
8 AFTA Technology

M. Charley Noecker, Jet Propulsion Laboratory (United States)

9 Starshade Technology

Stuart Shaklan, Jet Propulsion Laboratory (United States)

10 Astrometry

Olivier Guyon, Subaru Telescope, National Astronomical Observatory of Japan (United States) 